\title{
Pervaporation Characteristics in Removal of Benzene from Water through Polystyrene-Poly (Dimethylsiloxane) IPN Membranes
}

\author{
Tadashi Uragami $^{1,2}$, Iusaku Sumida ${ }^{1}$, Takashi Miyata ${ }^{1,2}$, Tadashi Shiraiwa ${ }^{1,2}$, Hiroshi Tamura ${ }^{1,2}$, \\ Tatsuo Yajima ${ }^{1,2}$

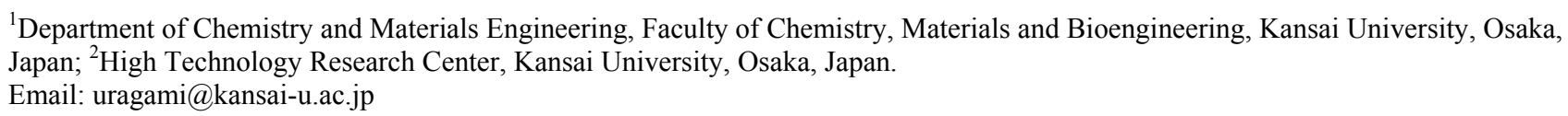

Received January $15^{\text {th }}, 2011$; revised February $1^{\text {st }}, 2011$; accepted February $6^{\text {th }}, 2011$.

\begin{abstract}
This paper focuses on the effects of the PSt content of polystyrene (PSt)-poly (dimethylsiloxane) (PDMS) interpenetrating network (IPN) polymer membranes on the pervaporation (PV) characteristics during the removal of benzene from an aqueous solution of dilute benzene. When an aqueous solution of $0.05 w t \%$ benzene was permeated through the PSt-PDMS IPN membranes, they showed high benzene/water selectivity. Both the permeability and the benzene/water selectivity of the membranes were enhanced with increasing PSt content in the PSt-PDMS IPN membrane. The physicochemical mechanism of permeation and separation through the PSt-PDMS IPN membranes during PV is also discussed. The best normalized permeation rate, separation factor for benzene selectivity, and PV separation index of the PSt-PDMS IPN membrane were $1.27 \times 10^{-6} \mathrm{kgm}\left(\mathrm{m}^{2} \mathrm{hr}\right)^{-1}, 3293$, and 41821 , respectively. These PV characteristics are discussed from the viewpoint of the chemical and physical structure of the PSt-PDMS IPN membranes.
\end{abstract}

Keywords: Interpenetrating Network Polymer, Polystyrene, Poly (Dimethylsiloxane), Pervaporation, Removal of Benzene

\section{Introduction}

Volatile organic compounds (VOCs) such as aromatic hydrocarbons and chlorinated hydrocarbons are used as washing solvents in the chemical industry, and cause soil pollution. Such pollution contaminates the subterranean water and consequently water in rivers and ponds. Drinking water sources are often dependent on this contaminated water. Furthermore, VOCs are known to be chemical materials that disturb internal secretions (environmental hormones), and are thus potentially dangerous to humans. As such, these chemicals have to be removed from contaminated water. An activated carbon method, ozone processing and other methods have been developed to remove VOCs from water [1]. However, these methods do not efficiently remove VOCs and the cost of treatment is high.

Pervaporation (PV) is a promising membrane technique for the separation of VOCs/water mixtures [2-8], as $\mathrm{PV}$ can be used to separate organic liquid mixtures such as azeotropic mixtures or close-boiling point mixtures. Poly(dimethylsiloxane) (PDMS) membranes have been studied as VOC-permselective membranes, because they have both high permselectivity and high permeability for organic chemicals in water [9-12]. The removal of chloroform in tap water through a hollow-fiber PDMS membrane, which showed high chloroform-permselectivity and permeability was demonstrated [13]. The high VOCspermselectivity of the PDMS membrane is attributable to its stronger affinity for VOCs than for water. Another advantage of the PDMS membrane is the high diffusivity of VOCs in the membrane due to its low $T \mathrm{~g}$. The PDMS membrane has some disadvantages however, most notably its weak mechanical strength. Nakagawa et al. introduced cross-linking into the PDMS membrane to improve its mechanical strength. The introduction of crosslinking however, led to a lower diffusivity of the permeants in the membrane [14].

Polymer blending is a promising technique for improving the physical and chemical properties of various 
materials. However, in general, the blending of general polymers is difficult because there are few combinations of miscible polymers. Since phase separation generally takes place in the blending of immiscible polymers, an improvement of the physical and chemical properties of a material is rarely achieved.

One of the methods to blend various polymers is the formation of an interpenetrating polymer network (IPN), which is defined as a combination of two polymers in network form $[15,16]$. IPNs have attracted our attention, since an improvement in the materials properties can be expected. Therefore, IPNs containing PDMS as a component were synthesized and their properties have been investigated extensively [17-20]. He et al. [17] obtained PDMS-poly(methyl methacrylate) (PMMA) IPNs by an in situ sequential synthesis and examined the properties of the resulting IPNs. They reported that interpenetrating the PMMA networks in the PDMS networks can improve the poor mechanical properties of the PDMS membranes. The structure of the PDMS component in the PDMS/ PMMA IPN which is the first-formed network, and constitutes a continuous phase. As such, the morphology of the membrane significantly affects the membrane its performance, and thus the morphology must be controlled by the volume fraction of each component, the preparation conditions, etc.

This paper focuses on the effects of the structure of IPN membranes composed of polystyrene (PSt), which is one of hydrophobic polymers, and PDMS on the removal of benzene from an aqueous solution of dilute benzene through their IPN membranes during PV. The relationship between the removal characteristics for the benzene/water selectivity and the structure of IPN membrane is discussed in detail.

\section{Experimental}

\subsection{Materials}

Hydroxyl-terminated PDMS base polymer (weight-average molecular weight was 20000 ), was supplied by Toray Dow Corning Silicons Co. Ltd. Tetraethoxysilane (TEOS) and stannous dibutyldilaurate were employed as a cross-linker and a catalyst in the polycondensation reaction of the PDMS base polymer, respectively. Benzoyl peroxide (BPO) as an initiator in the bulk-polymerization of styrene (St) was purified by reprecipitation from chloroform into methanol. Divinylbenzene (DVB) was used as a cross-linker in the bulk-polymerization of styrene. All other solvents and reagents were of analytical grade from commercial sources, and were used without further purification.

\subsection{Preparation of PSt-PDMS IPN Membranes}

PSt-PDMS IPN membranes were prepared by the polycondensation of the PDMS base polymer and the bulkpolymerization of St as follows: a mixture of the PDMS base polymer, TEOS (excess to the PDMS base polymer), and stannous dibutyldilauraete $(4.0 \mathrm{wt} \%$ relative to the PDMS base polymer) for the formation of the PDMS network was mixed with a mixture of the St monomer, $\mathrm{DVB}$, and $\mathrm{BPO}(0.3 \mathrm{wt} \%$ relative to the $\mathrm{St}$ monomer) for the formation of the PSt network. Two glass plates with a silicon sheet were separated by a $100 \mu \mathrm{m}$ of spacer to form a mold. The above mixture was then transferred into the mold. At first, the PDMS network was formed at $25^{\circ} \mathrm{C}$ for $8 \mathrm{~h}$. Then, the temperature was raised to $80^{\circ} \mathrm{C}$, and the $\mathrm{St}$ monomer was bulk-polymerized for $8 \mathrm{~h}$ under a nitrogen atomosphere in situ. The resulting PSt-PDMS IPN membranes were sufficiently washed in $n$-hexane to completely remove the unreacted St monomer and PDMS base polymer which had not interpenetrated, the St homopolymer and other reagents. The PSt-PDMS membranes were dried sufficiently in vacuo, and their compositions determined by elementary analysis.

\subsection{Glass Transition Temperature (Tg) Measurements}

The glass transition temperatures (Tgs) of the dry PStPDMS IPN membranes were determined by differential scanning calorimetry (DSC) (Rigaku, TAS-200). The specimens were heated from about $-150^{\circ} \mathrm{C}$ to $+159^{\circ} \mathrm{C}$ at a heating rate of $20^{\circ} \mathrm{C} / \mathrm{min}$.

\subsection{Transmission Electron Microscope (TEM)}

The PSt-PDMS IPN membranes were vapor-stained with an aqueous solution of $0.5 \mathrm{wt} \% \mathrm{RuO}_{4}$ in glass-covered dishes [21]. The stained membranes were embedded in epoxy resin and cross-sectioned into thin films using a microtome (Leica; Reichert Ultracut E). The resin morphological features of the stained membranes were observed with a transmission electron microscope (TEM) (JEOL JEM-1210) at an accelerating voltage of $80 \mathrm{kV}$.

\subsection{Contact Angle Measurements}

The contact angles of water on the surface of the PStPDMS IPN membranes were measured using a contact angle meter (Erma model G-1) at $25^{\circ} \mathrm{C}$. The contact angle, $\theta$, was determined from the advancing contact angle, $\theta_{a}$, and the receding contact angle, $\theta_{r}$, by Equation (1):

$$
\theta=\cos ^{-1}\left[\frac{\left(\cos \theta_{a}+\cos \theta_{r}\right)}{2}\right]
$$

where $\theta_{a}$ and $\theta_{r}$ are the advancing contact angle and the 
receding contact angle, respectively.

\subsection{Composition of Solution Sorbed into Membrane}

Dry PSt-PDMS IPN membranes were immersed into an aqueous solution of benzene in a sealed vessel at 30,40 , 50 and $60^{\circ} \mathrm{C}$ for $24 \mathrm{hr}$. Next, membranes swollen with this feed solution were placed into a reduced pressure system, and the benzene/water solution sorbed into the membrane was completely vaporized by heating and mem-brane was completely vaporized by heating and trapped by liquid nitrogen. The benzene/water composition was then determined by a gas chromatograph (Yanaco G2700) using a total carbon detector (TCD) plus a capillary column (Yanaco Co. Ltd: Porapak P and Q) heated to $150^{\circ} \mathrm{C}$. The composition of benzene/water in the PStPDMS IPN membranes and in the feed solution yielded the sorption selectivity, $\alpha_{\text {sorp. } \mathrm{Bz} / \mathrm{H}_{2} \mathrm{O}}$, as expressed in Equation (2):

$$
\alpha_{\text {sorp. Bz } / \mathrm{H}_{2} \mathrm{O}}=\left(M_{\mathrm{Bz}} / M_{\mathrm{H}_{2} \mathrm{O}}\right) /\left(F_{\mathrm{Bz}} / F_{\mathrm{H}_{2} \mathrm{O}}\right)
$$

where $F_{\mathrm{Bz}}$ and $F_{\mathrm{H}_{2} \mathrm{O}}$ are the weight fractions of benzene and water in the feed solution and $M_{\mathrm{Bz}}$ and $M_{\mathrm{H}_{2} \mathrm{O}}$ are those in the membrane, respectively.

\subsection{Membrane Density}

The density of the PSt-PDMS IPN membranes was dtermined by measuring their weights in air and methanol with an electric gravity meter (Mirage Boeki, SD-120L) at $25^{\circ} \mathrm{C}$.

\subsection{Cross-Linking Density}

The cross-linking density, $\rho$, of the PSt-PDMS IPN membranes was calculated based on the network theory of rubber elasticity given in Equation (3):

$$
\rho=\frac{E^{\prime}}{3 d \phi R T}
$$

where $E^{\prime}$ is determined from measurements with a dynamic mechanical analyzer (Rheogel-E4000 F3, UBM) under the following conditions: frequency, 1, 2, 4, $10 \mathrm{~Hz}$; temperature, $40^{\circ} \mathrm{C}$; and $d$ is the membrane density, $d$ is the front factor (where $\phi=1$ ), $R$ is the gas constant and $T$ is the absolute temperature.

\subsection{Permeation Measurements}

The PV apparatus and cell (GTR-tech Co. Ltd: GTR10VAK, G2700TF) used in this study are shown in Figure 1. The PV experiments were performed by operating the five electronic magnetic valves A $\sim \mathrm{D}$ in the PV apparatus as reported in a previous work [22]. The conditions

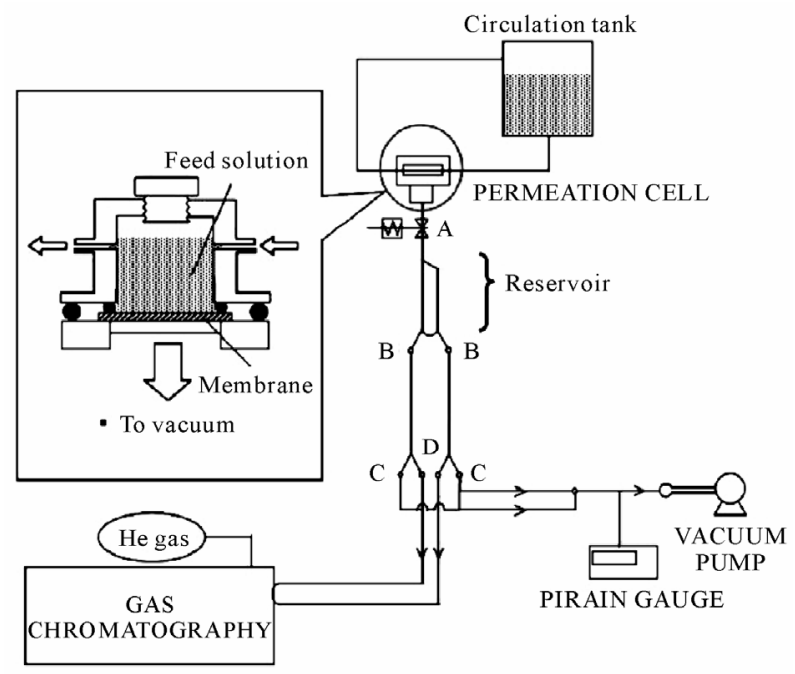

Figure 1. Pervaporation apparatus and permeation cell (GTR-tech. Co.). Five electronic magnetic valves (A through D) in the pervaporation apparatus are switched automatically by a given setting.

of the PV experiment were as follows: effective membrane area, $15.2 \mathrm{~cm}^{2}$; and permeation temperatures, 30 , 40,50 , and $60^{\circ} \mathrm{C}$. The feed solutions were aqueous solutions of dilute benzene. The feed solution was circulated between the PV cell and the feed tank to maintain a constant concentration of the feed solution in the PV cell. The permeation rate $\left[\mathrm{kg} /\left(\mathrm{m}^{2} \mathrm{~h}\right)\right]$ for an aqueous benzene solution during PV was determined from the weight $(\mathrm{kg})$ of the collected permeate, the permeation time (h), and the effective membrane area $\left(\mathrm{m}^{2}\right)$. In order to facilitate a comparison of the permeation rates of membranes of different thicknesses, the normalized permeation rate (NPR) $\left[\mathrm{kgm}\left(\mathrm{m}^{2} \mathrm{~h}\right)^{-1}\right]$, which is the product of the permeation rate and the membrane thickness, was used. The results for the permeation of aqueous solutions by PV were reproducible, and the errors inherent in these permeation measurements ranged within a few percent for the permeation rates through the membranes.

The benzene concentration in the permeate was also determined by a gas chromatograph (Yanaco G2700) using a total carbon detector (TCD) plus a capillary column (Yanaco Co. Ltd: Porapak P and Q) heated to $150^{\circ} \mathrm{C}$.

The separation factor, $\alpha_{\text {sep.Bz } / \mathrm{H}_{2} \mathrm{O}}$, was calculated from Equation (4):

$$
\alpha_{\text {sep.Bz } / \mathrm{H}_{2} \mathrm{O}}=\frac{\left(P_{\mathrm{Bz}} / P_{\mathrm{H}_{2} \mathrm{O}}\right)}{\left(F_{\mathrm{Bz}} / F_{\mathrm{H}_{2} \mathrm{O}}\right)}
$$

where $F_{\mathrm{Bz}}$ and $F_{\mathrm{H}_{2} \mathrm{O}}$ are the weight fractions of benzene and water in the feed solution, and $P_{\mathrm{Bz}}$ and $P_{\mathrm{H}_{2} \mathrm{O}}$ are 
those in the permeate, respectively.

\section{Results and Discussion}

\subsection{Characterization of PSt-PDMS IPN Membranes}

Table 1 shows the composition of the PDMS-PDMS IPN membranes determined by elemental analysis, and the glass transition temperature ( $T \mathrm{~g}$ 's) of the PSt-PDMS IPN membranes determined by DSC. The PSt content in the membrane was lower than that in the feed. This is due to the fact that not all of the St monomer was polymerized, and the PSt chains which did not interpenetrated into the PDMS networks were extracted by benzene during the process of the purification of the PSt-PDMS IPN membranes. This hypothesis is supported by the fact that the PSt chains which interpenetrated into the PDMS networks were not dissolved by benzene. As can be seen in Table 1, all of the PSt-PDMS IPN membranes have two $T$ g's at about 82.2 and $-118.7^{\circ} \mathrm{C}$. The $T \mathrm{~g}$ 's of the PSt and PDMS homopolymer membranes were 82.5 and $-118.7^{\circ} \mathrm{C}$, respectively. The higher and lower $T \mathrm{~g}$ 's can be assigned to the PSt and PDMS component, respectively. These two Tg's in the PSt-PDMS IPN membranes suggest that the PSt-PDMS IPN membranes could be formed by heterogeneous structures consisting of a PSt phase and a PDMS phase.

The TEM images for the cross sections of the PStPDMS IPN membranes with different compositions are shown in Figure 2. The image contrasts results due to the difference in the electron density of the silicon and carbon atom. The white and dark part in Figure 2 are due to the PSt and PDMS phase, respectively. The TEM obser-

Table 1. Compositions and glass transition temperatures (Tg's) of the Pst-PDMS IPN membranes.

\begin{tabular}{cccc}
\hline \multicolumn{4}{c}{ PSt-PDMS IPN Membrane } \\
\hline $\begin{array}{c}\text { PSt content in } \\
\text { Feed (mol\%) }\end{array}$ & $\begin{array}{c}\text { PSt content in } \\
\text { Membrane (mol\%) }\end{array}$ & High & low \\
\hline 0 & 0 & - & -118.8 \\
10 & 3.8 & 82.8 & -118.2 \\
20 & 12.7 & 83.8 & -117.9 \\
30 & 19.6 & 81.9 & -119.1 \\
40 & 26.5 & 83.1 & -119.4 \\
50 & 34.2 & 80.3 & -118.0 \\
70 & 54.5 & 81.5 & -120.1 \\
100 & 100 & 82.5 & - \\
\hline
\end{tabular}

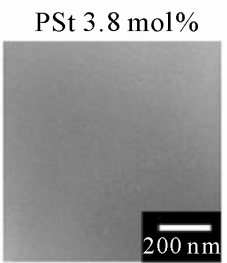

(a)

PSt $26.5 \mathrm{~mol} \%$

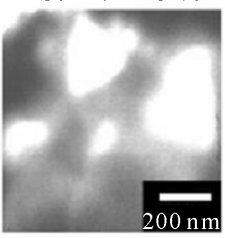

(d)

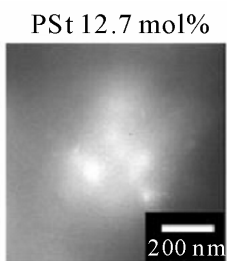

(b)

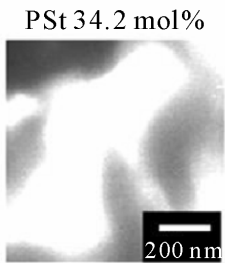

(e)

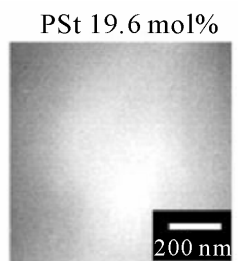

(c)

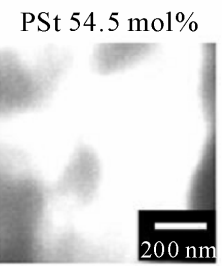

(f)
Figure 2. TEM images of the cross-section of PSt-PDMS IPN membranes.

vations demonstrated that the PSt-PDMS IPN membranes have microphase-separated structures. With increasing PSt content as the PSt content (Figure 2(b-d)) from low to high, the PSt phase clearly increased, and every PSt-PDMS IPN membrane had a morphology in which the PSt domain exists in the PDMS matrix. When the PSt content was $55 \mathrm{~mol} \%$ (Figure 2(f), however, the continuous phase of the PDMS component disappeared. In our previous studies, the morphology of graft- and block-copolymer membranes consisting of methylmethacrylate (MMA) and dimethylsiloxane macromonomer (DMS) was observed by TEM $[23,24]$. These PMMA-gPDMS and PMMA- $b$-PDMS membranes had the microphase-separated structures with continuous phases. However, the morphology of the PSt-PDMS IPN membranes was significantly different from those of the PMMA-gPDMS and PMMA- $b$-PDMS membranes One difference in these morphologies can be attributed to the difference between the blend versus graft or block polymers. The PSt-PDMS IPN membranes were prepared by the bulkpolymerization of St in the PDMS networks. Therefore, the PSt components are liable to form large domains in the continuous PDMS phase, and have difficulty in forming a continuous phase. This is the reason why the PSt-PDMS IPN membranes have the microphase-separated structures consisting of the PSt domain and the continuous PDMS phase as shown in Figure 2.

\subsection{Permeation and Separation Characteristics through PSt-PDMS IPN Membranes}

Figure 3 shows the effect of PSt content in IPN on the normalized permeation rate and benzene concentration in the permeate for an aqueous solution of $0.05 \mathrm{wt} \%$ benzene through PSt-PDMS IPN membranes during PV. As 


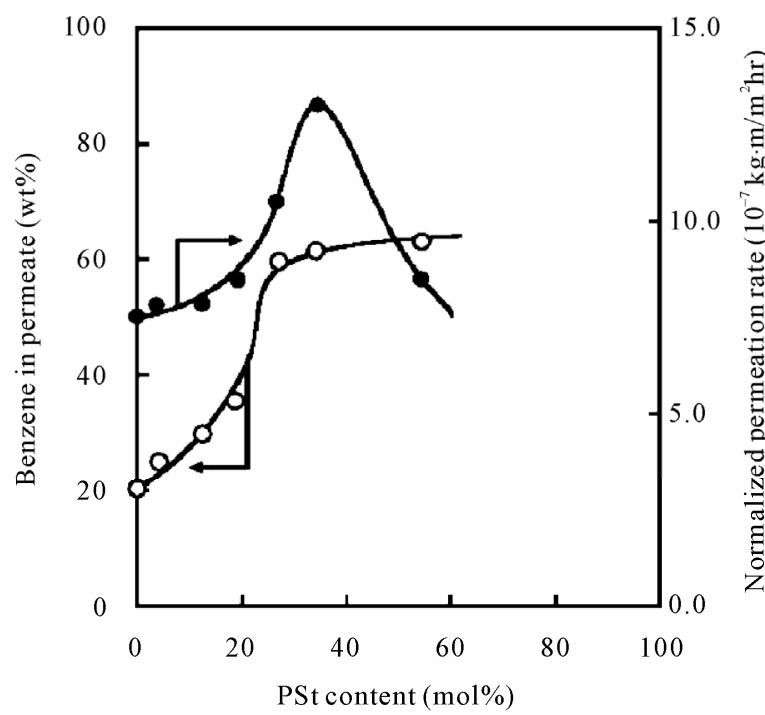

Figure 3. Effects of the PSt content on the normalized permeation rate $(\bullet)$ and the benzene concentration in the permeate $(\circ)$ for an aqueous solution of $0.05 \mathrm{wt} \%$ benzene during PV.

can be seen from Figure 3, The normalized permeation rate increased with increasing PSt content in IPN but decreased over $40 \mathrm{~mol} \%$ of PSt. With increasing PSt content, the benzene concentration in the permeate increased. The results until $40 \mathrm{~mol} \%$ of PSt indicate that the increase of PSt content in IPN membrane can enhance both permeation rate and benzene/water selectivity. In general, most modification of PV membranes cannot improve permselectivity without lowering permability. Therefore, it is unusual that the increase of PSt content in IPN membrane enables the membrane selectivity to increase without lowering the permeability.

\subsection{Chemical Structure of PSt-PDMS IPN Membranes}

In general, the permeation and separation characteristics of organic liquid mixtures through polymer membranes during PV are based on the solubility of the permeants into the polymer membrane (sorption process), and the diffusivity of the permeants in the polymer membrane (diffusion process). The solubility and diffusivity of the permeants are significantly influenced by the chemical and physical structures of the polymer membranes. Therefore, we characterized the PSt-PDMS IPN membranes from the viewpoint of the chemical and physical structures of the polymer membranes. First, in order to discuss the effects of the interpenetration of PSt into the PDMS matrix membrane for the sorption process, the contact angle for water on the surface of the PSt-PDMS IPN membranes was measured. Figure 4 shows the ef- fect of the PSt content on the contact angle for water, The contact angle for water on the surface of the PStPDMS IPN membrane decreased with increasing PSt content. In order to investigate the results from Figure 4 in detail, the solubility parameters of PSt and PDMS, which comprise the IPN matrix, with benzene and water, which are the permeants are summarized in Table 2. These solubility parameters were adopted from the solubility parameters of Hansen and were calculated from data of Fedors [25] using the following Equation (5):

$$
\delta=((\Delta H-R T) / T)^{1 / 2}=(\Delta E / V)^{1 / 2}
$$

where $\Delta H$ is the enthalpy change when dissolved, $\Delta E$ is the cohesive energy density and $V$ is the molar volume of the solvent. These results suggest that the surface of the PSt-PDMS IPN membranes became more hydrophilic, and that their affinity for water increased with increasing PSt content.

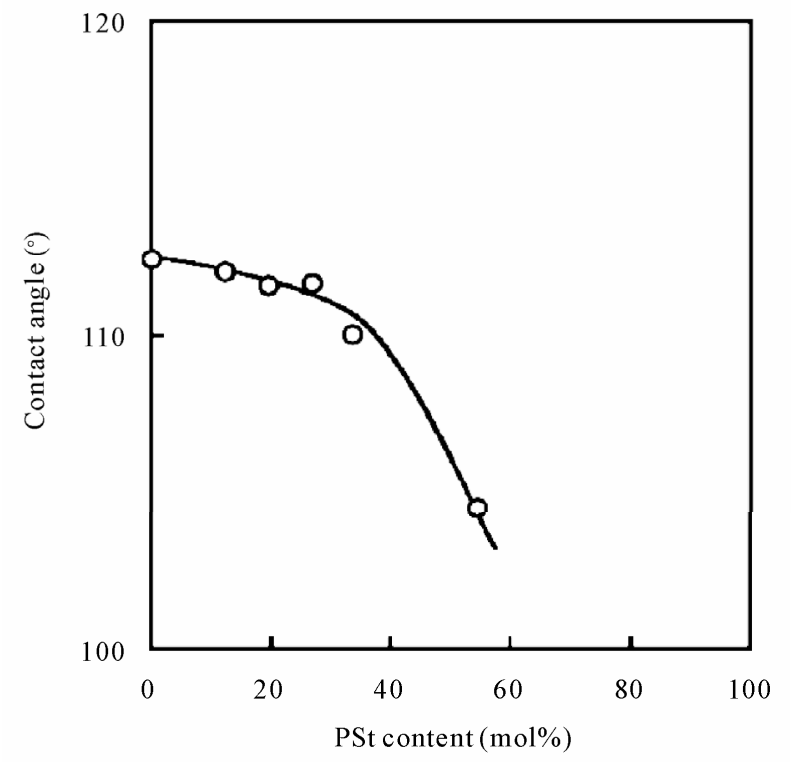

Figure 4. Contact angle for water of the surface of PStDMS IPN membranes as a function of their PSt content.

Table 2. Hansen solubility for benzene, water, PSt and PDMS.

\begin{tabular}{ccccc}
\hline \multirow{2}{*}{ Material } & \multicolumn{4}{c}{ Solubility parameter } \\
\cline { 2 - 5 } & $\delta_{d}$ & $\delta_{p}$ & $\delta_{h}$ & $\delta$ \\
\hline Benzene & 18.4 & 0 & 2.0 & 18.6 \\
Water & 15.5 & 16.0 & 42.4 & 47.9 \\
PSt & 19.2 & 0.9 & 2.1 & 19.2 \\
PDMS & 15.9 & 0.1 & 4.7 & 16.5 \\
\hline
\end{tabular}


The difference in the solubility parameter, $\Delta$, was calculated from the solubility parameter of benzene $\left(\delta_{\mathrm{Bz}}\right)$ or water $\left(\delta_{\mathrm{H}_{2} \mathrm{O}}\right)$ and the PSt component $\left(\delta_{\mathrm{PSt}}\right)$ or PDMS component $\left(\delta_{\mathrm{PDMS}}\right)$ which are consisting of the IPN membrane matrix and using Equations (6-9), respecttively.

$$
\begin{gathered}
\Delta=\left|\delta_{\mathrm{PSt}}-\delta_{\mathrm{Bz}}\right| \\
\Delta=\left|\delta_{\mathrm{PSt}}-\delta_{\mathrm{H}_{2} \mathrm{O}}\right| \\
\Delta=\left|\delta_{\mathrm{PDMS}}-\delta_{\mathrm{Bz}}\right| \\
\Delta=\left|\delta_{\mathrm{PDMS}}-\delta_{\mathrm{H}_{2} \mathrm{O}}\right|
\end{gathered}
$$

A small $\Delta$ means that the PSt or PDMS component of PSt and PDMS in the PSt-PDMS IPN membrane has a strong affinity for benzene or water. From Table 2, it is clear that the PSt components have a higher affinity for water and benzene than the PDMS components. Therfore, as shown in Figure 4, it is easily understood the with increasing PSt content, the affinity for water of the PSt-PDMS IPN membranes increased, and consequently the contact angle for water decreased.

\subsection{Benzene/Water Selectivity in Sorption Process}

The enthalpy for the sorption process can be determined by measuring the temperature dependency and using Equation (10):

$$
\begin{aligned}
\Delta H_{\text {sorp }} & =\ln \left(P / P_{0}\right) / R T \\
P & =\alpha_{\text {sorp }} L \Delta C
\end{aligned}
$$

where $\Delta H_{\text {sorp }}, P, P_{0}, L \Delta C, T$, and $R$ are the enthalpy for the sorption, the normalized permeation rate, the correction coefficient, the membrane thickness, the concentration difference, the absolute temperature and the gas constant, respectively. $\alpha_{\text {sorp }}$ is defined as expressed in Equation (2).

Figure 5 shows the sorption selectivity, $\alpha_{\text {sorp. Bz } / \mathrm{H}_{2} \mathrm{O}}$, and the enthalpy for the sorption, $\Delta H_{\text {sorp }}$, as a function of the PSt content in the PSt-PDMS IPN membranes. As one can see from Figure 5, with increasing PSt content, the sorption selectivity for benzene/water increased and the enthalpy for the sorption decreased, and these increases and decreases were especially remarkable at over $35 \mathrm{~mol} \%$ of PSt content. The decrease in the enthalpy for the sorption could be attributed to the fact that the affinity for benzene of the PSt-PDMS IPN membranes with increased in the PSt content also increased. Consequently, this decrease in the enthalpy for the sorption steers the increase in the sorption selectivity for benzene/water.

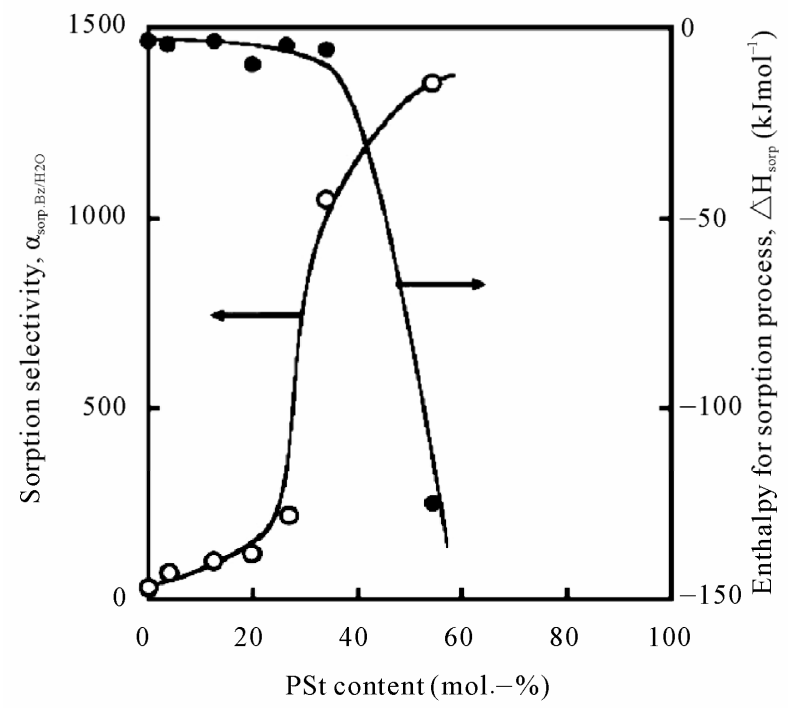

Figure 5. Effects of the PSt content on the enthalpy for the sorption process, $\Delta \mathbf{H}_{\text {sorp. }}(\bullet)$ and the sorption selectivity, $\Delta_{\text {sorp.Bz/H2O }}(\circ)$.

\subsection{Physical Structure of PSt-PDMS IPN Membranes}

It is well-known that the chemical structure of a polymer membrane significantly affects the sorption of the permeants into the membrane in the sorption process and the physical structure strongly influences the diffusion of the permeants into the membrane during the diffusion process [26]. Next, the physical structure of the PSt-PDMS IPN membranes was investigated. Figure 6 shows the effect of the PSt content on the cross-linking density of the PSt-PDMS IPN membranes. The cross-linking density and the degree of swelling increased with increasing PSt content. In particular, the PSt-PDMS IPN membrane containing PSt of about $55 \mathrm{~mol} \%$ had a high cross-linking density. This increase in the cross-linking density with increasing PSt content suggests the formation of a PSt-PDMS IPN membrane with a denser network structure. The highest density membrane was observed at 55 mol\% PSt, which does not have a continuous phase of the PDMS component.

\subsection{Energy State on Each Process during Permeation through PSt-PDMS IPN Membranes}

In order to physicochemically interpret the permeation state through the PSt-PDMS IPN membranes, the activation energy of the permeation process was determined by Equation (11):

$$
P=P_{0} \exp \left(-E_{p} / R T\right)
$$




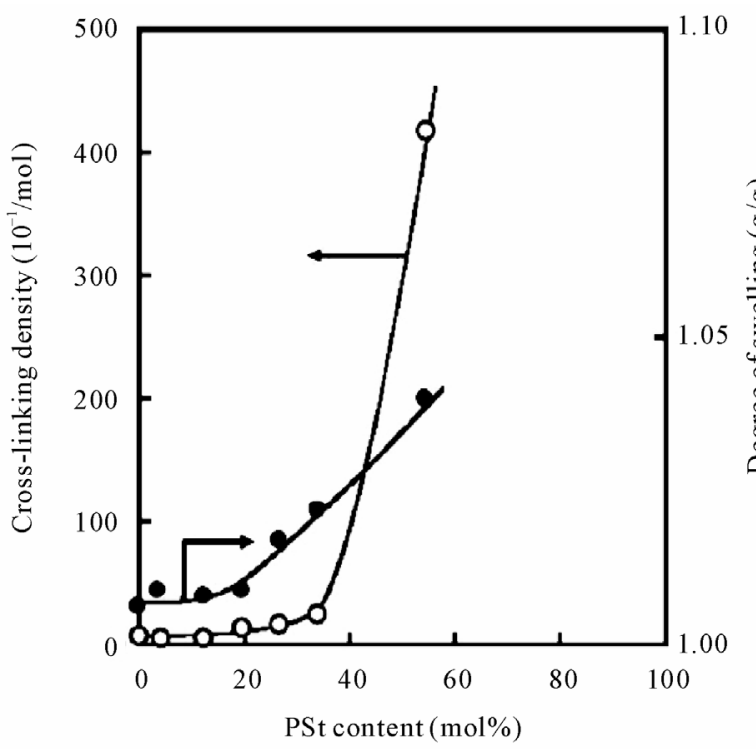

Figure 6. Effects of the PSt content on the cross-linking density of PSt-PDMS IPN membranes.

where $E_{p}$ is the activation energy for the permeation through the membrane.

In Figure 7, the activation energy for the permeation of an aqueous solution of $0.05 \mathrm{~mol} \%$ benzene though the PSt-PDMS IPN membranes is shown as a function of the PSt content. The activation energy for the permeation increased with increasing PSt content in the PSt-PDMS IPN membranes. In order to determine the course of this increase in the activation energy, the effects of the PSt content on the enthalpy for the sorption of the permeants into the PSt-PDMS IPN membranes on the sorption process and the activation energy for the diffusion of the permeants into the PSt-PDMS IPN membranes were determined by Equation (12):

$$
E_{p}=\Delta H+E_{\text {diff }}
$$

where $E_{\text {diff }}$ is the activation energy for the diffusion process.

Figure 8 shows the sorption enthalpy of the sorption process and the activation energy of the diffusion process show as a function of the PSt content. The sorption enthalpy decreased as a first order function, and at over 35 mol\% of PSt content this decrease became remarkable. The decrease in these enthalpies could be attributed to the fact that the affinity for benzene increased with the introduction of the PSt and DVB that comprise aromatic networks. This remarkable decrease in the sorption enthalpy was also due to the fact that the membrane surface was occupied by the PSt components when the membrane composition was over $50 \mathrm{~mol} \%$.

On the other hand, the activation energy of the diffu-

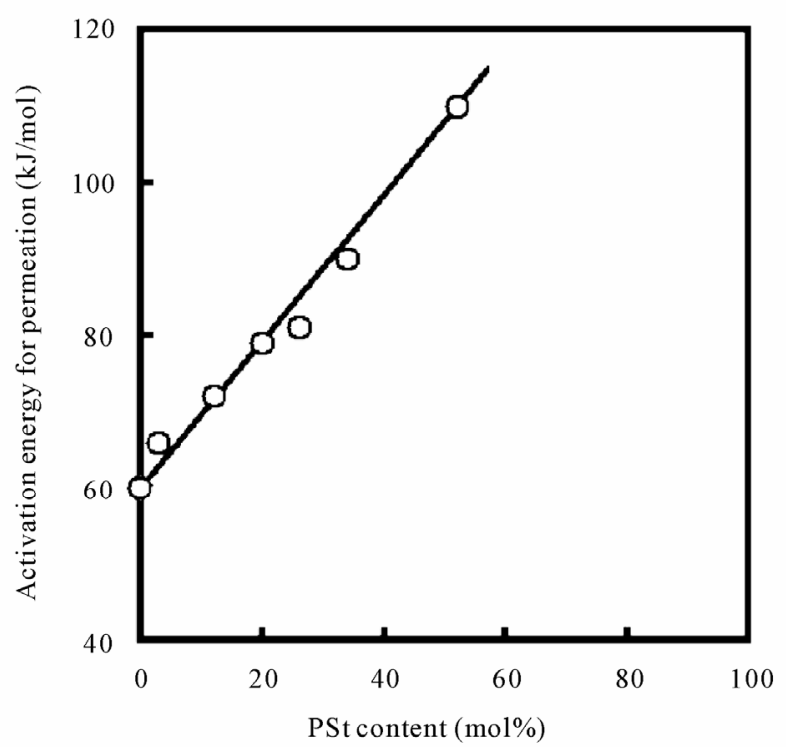

Figure 7. Relationship between the PSt content in PStPDMS IPN membrane and the activation energy for permeation through PSt-PDMS IPN membranes during PV.

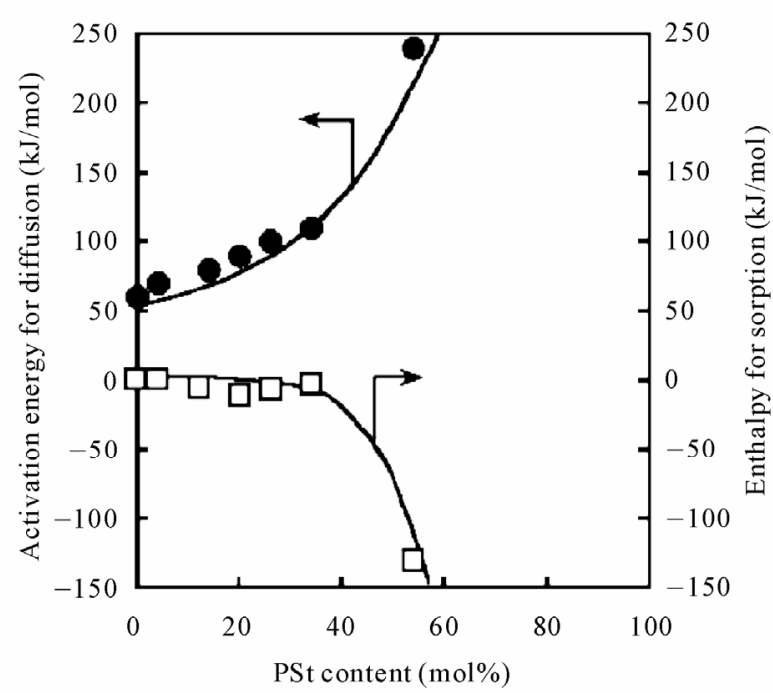

Figure 8. Effect of the PSt content in PSt-PDMS IPN membranes on the sorption enthalpy of the sorption process $(\square)$ and the activation energy on the diffusion process $(\bullet)$.

sion process increased as a first order function and this increase at over $35 \mathrm{~mol} \%$ of PSt was significant. An investigation of the phase structure of the PSt-PDMS IPN membrane as mentioned above could explain these significant increases in activation energy for the membrane with a disappearance of the continuous phase of the PDMS component. Until a PSt content of $35 \mathrm{~mol} \%$, with increasing PSt content the activation energy for the diffusion process increased with an increase in the domain 
of the PSt component. This result suggests that a continuous structure and the size of the PDMS domain significantly influence on the diffusivity of permeants. It was concluded that the benzene/water selectivity can be improved by controlling the continuous structure and size of the PDMS domain.

\subsection{Permselectivity Mechanism of PSt-PDMS IPN Membranes}

In the solution-diffusion model [27-31], differences in the solubility of the permeants in polymer membranes and differences in the diffusivity of the permeants in polymer membranes are very significantly related to the permselectivity [22]. It is very important to determine the sorption selectivity and diffusion selectivity to elucidate the separation mechanism of a dilute aqueous solution of benzene through the PSt-PDMS IPN membranes. Thus, in order to discuss the benzene permselectivity of a dilute aqueous solution of benzene through the PSt-PDMS IPN membranes from the viewpoint of the solution-diffusion mechanism, both the sorption selectivity and the diffusion selectivity must be determined. The sorption selectivity, $\alpha_{\text {sorp. } B z / H_{2} O}$, was determined from Equation (2) and the diffusion selectivity, $\alpha_{\text {diff } B z / H_{2} O}$, can be calculated from Equation (13) using Equations (2) and (4),

$$
\alpha_{\text {diff } \cdot B z / H_{2} O}=\alpha_{\text {sorp } . B z / H_{2} O}
$$

In Figure 9, the separation factor, the sorption selectivity and the diffusion selectivity for an aqueous solution of $0.05 \mathrm{~mol} \%$ benzene through the PSt-PDMS IPN membranes are shown as a function of the PSt content. The sorption selectivities of membranes with various PSt contents were all greater than their diffusion selectivities. This observation suggests that the removal of benzene from a dilute aqueous solution of benzene using the PStPDMS IPN membranes is mainly governed by the sorption process. The fact that benzene was preferentially absorbed into these membranes rather than water also supports this conclusion. Furthermore, the sorption selectivity increased with increasing PSt content. The increase in the benzene permselectivity with an increased PSt content was due to an increase in the solubility of benzene in the PSt-PDMS IPN membranes.

\subsection{Estimation of PSt-PDMS IPN Membrane Performance}

In Table 3, the pervaporation separation index (PSI) [22], which is the product of the permeation rate and the separation factor, for an aqueous solution of $0.05 \mathrm{wt} \%$ benzene through the PSt-PDMS IPN membranes (PV conditions: permeation temperature, $40^{\circ} \mathrm{C}$; pressure of the permeation side, $1.33 \mathrm{~Pa}$ ), was used as a measure of membrane per-
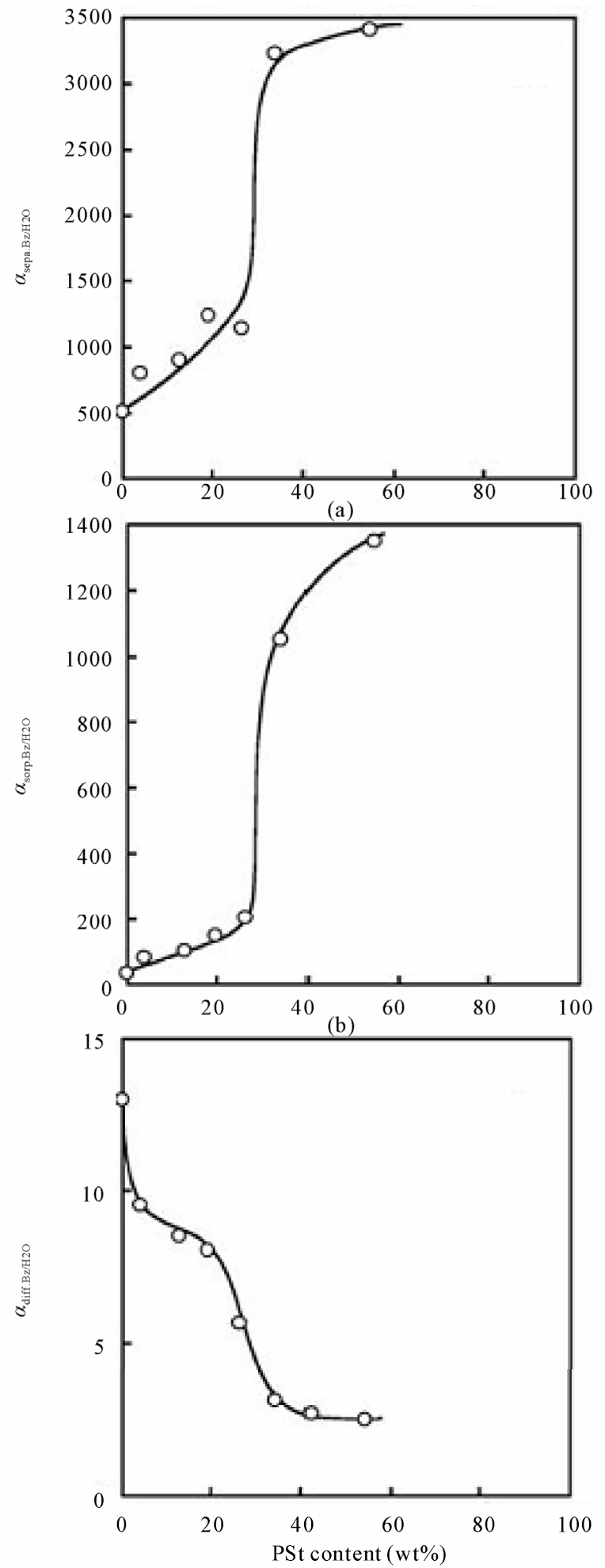

(c)

Figure 9. Effects of the PSt content on the separation factor (a), the sorption selectivity (b) and the diffusion selectivity (c) for an aqueous solution of $0.05 \mathrm{wt} \%$ benzene through PSt-PDMS IPN membranes during PV. 
Table 3. Membrane perfomance of PSt-PDMS IPN membranes.

\begin{tabular}{cccc}
\hline $\begin{array}{c}\text { PSt Content in } \\
\text { Membrane } \\
(\mathrm{mol} \%)\end{array}$ & $\begin{array}{c}\text { Separation } \\
\text { Factor }\end{array}$ & $\begin{array}{c}\text { Normalized } \\
\text { Permeation Rate } \\
\left(10^{-7} \mathrm{kgm}\left(\mathrm{m}^{2} \mathrm{~h}\right)^{-1}\right)\end{array}$ & PSI \\
\hline 0 & 479 & 6.99 & 3348 \\
3.8 & 751 & 7.53 & 5655 \\
12.7 & 1750 & 8.19 & 14332 \\
19.6 & 1996 & 9.17 & 18303 \\
26.5 & 2926 & 10.1 & 29552 \\
34.2 & 3293 & 12.7 & 41821 \\
54.5 & 3563 & 8.45 & 30107 \\
\hline
\end{tabular}

formance during PV. As can be seen in Table 3, both the normalized permeation rate and the separation factor for the benzene/water selectivity of PSt-PDMS IPN membranes were improved with increasing PSt content. Based on this result, we discovered that the introduction of the PSt component into the IPN membrane matrix using a suitable component with a high affinity for a permeant is a very effective method to give both a high permeation rate and high permselectivity.

\section{Conclusions}

The mechanism of permeation and separation for an aqueous solution of dilute benzene through PSt-PDMS IPN membranes could be explained by the model illustration shown in Figure 10. For the separation characteristics for an aqueous solution of dilute benzene through the PSt-PDMS IPN membranes, with an increasing PSt content in the PSt-PDMS IPN membrane, the benzene molecule was predominantly incorporated into the PStPDMS IPN membranes with a decrease in the sorption enthalpy during the sorption process. Consequently the PSt-PDMS membranes showed an excellent benzene/ water selectivity. A PSt-PDMS IPN membrane containing $55 \mathrm{~mol} \%$ of PSt showed the highest benzene selectivity. On the other hand, in the term of permeation characteristics, the permeability was increased by an increase in the degree of swelling of the PSt-PDMS IPN membrane and the formation of a continuous phase of the PDMS component until $35 \mathrm{~mol} \%$ PSt content. A PSt-PDMS IPN membrane with a PSt content of $55 \mathrm{~mol} \%$ had the highest degree of swelling but the permeability was decreased by a decrease in the diffusivity of the permeants, because the continuous phase of the PDMS component disappeared. It is noted that the permeation and separation behavior of the PST-PDMS IPN membranes was dependent on the continuity of the PDMS phase in the PSt-PDMS IPN membranes, and significantly influenced the permeability. On the basis of the above results, it is suggested that an excellent benzene permeable mem-

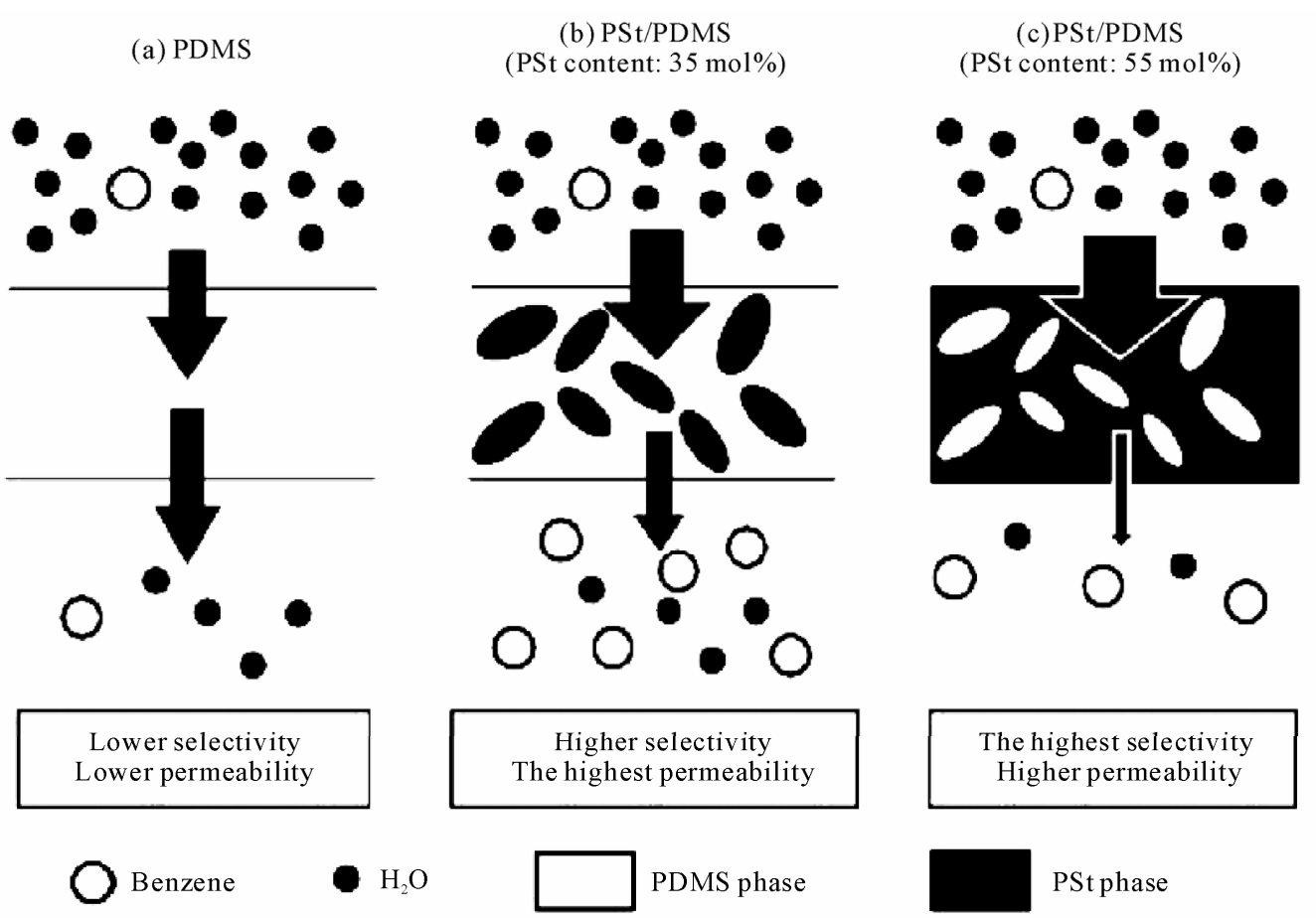

Figure 10. Tentative mechanism of the permeation and separation of an aqueous solution of dilute benzene though PSt-PDMS IPN membranes during PV. 
brane can be prepared by controlling both the continuous phase and the domain size of the PDMS component.

\section{Acknowledgements}

This work was financially supported by the Kansai University Special Research Fund, 2008 and by the 'HighTech Research Center"' Project in Matching Fund Subsidy for Private Universities, 2005-2009.

\section{REFERENCES}

[1] R. W. Baker, "Pervaporation Process," In: R. W. Baker, E. L. Cussler, W. Eykamp, W. J. Koros and H. Strathmann, Eds.; Membrane Separation System, Recent developments and Future Directions, Noyes Data Corp, Park Ridge, 1991, p. 151.

[2] W. W. Lau, J. Finlayson, J. M. Dickson, J. Jian and M. A. Brook, "Pervaporation Performance of OligosilylstyrenePolydimethylsiloxane Membrane for Separation of Organics from Water," Journal of Membrane Science, Vol. 134, No. 2, 1997, pp. 209-217. doi:10.1016/S0376-7388(97)00131-2

[3] T. A. C. Oliveria, J. T. Scarpello and A. G. Livinhston, "Pervaporation-Biological Oxidationhybrid Process for Removal of Volatile Organic Compounds from Wastewater," Journal of Membrane Science, Vol. 195, No. 1, 2002, pp. 75-88. doi:10.1016/S0376-7388(01)00555-5

[4] Q. L. Liu and H. Xiao, "Silicalite-Filled Poly (Siloxane Imide) Membranes for Removal of VOCs from Groundwater," Journal of Membrane Science, Vol. 230, 2004, pp. 121-129. doi:10.1016/j.memsci.2003.11.004

[5] F. Vilaplana, M. Osorio-Galindo, A. Iborra-Clar, M. Alcaina-Miranda and A. Ribes-Greus, "Swelling Behavior of PDMS-PMHS Pervaporation Membranes in Ethyl Acetate-Water Mixtures," Journal of Applied Polymer Science, Vol. 93, 2004, pp. 1384-1393. doi:10.1002/app.20596

[6] F. Heymes, P. M. Demoustier, F. Carbit, J. L. Fanlo and P. Moulin, "Recovery of Toluene from High Temperature Boiling Adsorbents by Pervspoprstion," Journal of Membrane Science, Vol. 284, 2006, pp. 145-154. doi:10.1016/j.memsci.2006.07.029

[7] H. F. Zhen, S. M. L. Jang W. K. Teo and K. Li, "Modified Silicone-PVDF Composite Hollow-Fiber Membrane Preparation and Its Application in VOC Separation," Journal of Applied Polymer Science, Vol. 99, No. 5, 2006, pp. 2497-2503. doi:10.1002/app.22860

[8] G. O. Yahara, "Separation of Volatile Organic Compounds (BTEX) from Aqueous Solutions by a Composite Organophilic Hollow Fiber Membrane-Based Pervaporation Process," Journal of Membrane Science, Vol. 319, No. 1-2, 2008, pp. 82-89. doi:10.1016/j.memsci.2008.03.024

[9] T. Miyata, J. Higuchi, H. Okuno and T. Uragami, "Preparation of Poly(Dimethylsiloxane)/Polystyrene Interpenetrating Polymer Network Membranes and Permeation of Aqueous Ethanol Solutions through The Mem- branes by Pervaporation," Journal of Applied Polymer Science, Vol. 61, 1996, pp. 1315-1324. doi:10.1002/(SICI)1097-4628(19960822)61:8<1315::AID -APP11>3.0.CO;2-Y

[10] T. Miyata, Y. Nakanishi and T. Uragami. "Ethanol Permselectivity of Poly(Dimethylsiloxane) Membranes Controlled by Simple Surface Modifications using Polymer Additives," Macromolecures, Vol. 30, No. 18, 1997, pp. 5563- 5565. doi:10.1021/ma9618843

[11] T. Uragami, T. Tsukamoto, K. Inui and T. Miyata, "Pervaporation Characteristics of a Benzoylchiotsan Membrane for Benzene/Cychlohexane Mixtures," Macromolecular Chemistry \& Physics, Vol. 199, 1998, pp. 49-54. doi:10.1002/(SICI)1521-3935(19980101)199:1<49::AIDMACP49>3.0.CO;2-B

[12] K. Inui, H. Okumura, T. Miyata and T. Uragami, "Permeation and Separation of Benzene/Cyclohexane Mixtures through Cross-Linked Poly(Alkyl Methacrylate) Membranes" Journal of Membrane Science, Vol. 132, No. 2, 1997, pp. 193-202. doi:10.1016/S0376-7388(97)00069-0

[13] P. R. Brooks and A. G. Livingstone, "Aqueous-Aqueous Extraction of Organic Pollutants through Tubular Silicone Rubber Membranes," Journal of Membrane Science, Vol. 104, No. 1-2, 1995, pp. 119-139. doi:10.1016/0376-7388(95)00020-D

[14] S. Mishima and T. Nakagawa, "Permselectivity of Volatile Organic Chlorinated Compounds through Modified Poly(Dimethylsiloxane) Membranes," Kobunnshi Ronbunshu, Vol. 54, 1997, pp. 375-383.

[15] D. Klempner and K. C. Frisch, "Polymer Alloys," Plenum Press, New York, 1977.

[16] L. H. Sperling, "Interpenetrating Polymeric Networks and Related Materials," Plenum Press, New York, 1981.

[17] X. W. He, J. M. Widmaier, J. E. Herz and G. C. Meyer, "Polydimethylsiloxane/Poly(Methylmethacrylate) Interpenetrating Polymer Networks 2. Synthesis and Properties," Polymer, Vol. 33, No. 4, 1992, pp. 866-871. doi:10.1016/0032-3861(92)90351-V

[18] J. S. Turner and Y. L. Cheng, "pH Dependence of PDMSPMMA IPN Morphology and Transport Properties," Journal of Membrane Science, Vol. 240, 2004, No. 1-2, pp. 19-24. doi:10.1016/j.memsci.2004.04.008

[19] A. Hillerstrom, M. Andersson, J. Pedersen, A. Altskar, M. Langton and J. van Stam, "Transparency and Wetability of PVP/PDMS-IPN Synthesized in Different Organic Solvents," Journalof Applied Polymer Science, Vol. 114, No. 3, 2009, pp. 1828-1839. doi:10.1002/app.30673

[20] R. Marques, T. MacLeod, I. Yoshida, V. Mno and M. Shiavon, "Synthesis and Characterization of Semi-Interpenetrating Networks Based on Poly(Dimethysiloxane) and Poly(Vinyl Alcohol)," Journal of Applied Polymer Science, Vol. 115, 2010, pp. 158-166. doi:10.1002/app.31006

[21] J. S. Trent, J. I. Scheinbeim, and P. R. Couchman, "Ruthenium Tetraoxide Staining of Polymers for Electron 
Microscopy," Macromolecures, Vol. 16, 1983, 1983, pp. 589-598.

[22] T. Uragami, S. Yamamoto and T. Miyata, (2003), "Dehydration from Alcohols by Polyioncomplex Cross-Linked Chitosan Composite Membranes during Evapomeation," Biomacromolecules, Vol. 4, No. 1, 2003, pp. 137-144. doi: $10.1021 / \mathrm{bm} 025642 \mathrm{o}$

[23] T. Uragami, H. Yamada and T. Miyata, "Removal of Dilute Volatile Organic Compounds (VOCs) in Water through Graft Copolymer Membranes Consisting of Poly (Alkylmethacrylate) and Poly(Dimethylsiloxane) by Pervaporation and Their Membrane Morphology," Journal of Membrane Science, Vol. 187, No. 1-2, 2001, pp. 255-269. doi:10.1016/S0376-7388(01)00355-6

[24] T. Uragami, T. Meotoiwa and T. Miyata, "Effects of the Addition of Calixarene Microphase-Separated Membranes for the Removal of Volatile Organic Compounds from Dilute Aqueous Solutions," Macromolecures, Vol. 34, No. 19, 2001, pp. 6806-6811.

[25] W. S. John, "Polymer Handbook," 3rd Edition, Elsevier, Amsterdam, 1989.

[26] T. Ohshima, M. Matsumoto, T. Miyata and T. Uragami, "Organic-Inorganic Hybrid Membranes for Removal of Benzene from an Aqueous Solution by Pervaporation,". Macromolecular Chemistry \& Physics, Vol. 206, No. 4, 2005, pp. 473-483. doi:10.1002/macp.200400401

[27] R. C. Binning, R. J. Lee, J. F. Jennings and E. C. Martin, "Separation of Liquid Mixtures by Permeation," Indus- trial Engineering Chemistry, Vol. 53, 1961, pp. 45-54. doi:10.1021/ie50613a030

[28] P. Aptel, J. Cuny, J. Jozefonvicz, G. Morel and J. Neel, "Liquid Transport through Membranes Prepared by Grafting of Polar Monomers onto Poly(Tetrafluoroethylene) Films. III. Steady Sate Distribution in Membrane during Pervaporation," Journal of Applied Polymer Science, Vol. 18, No. 2, 1974, pp. 365-398. doi:10.1002/app.1974.070180205

[29] E. E. Lee and A. L. Bunge, "Chemical Transport in Silicone Rubber Membranes from Pure Powders and Satulated Aqueous Solutions," Journal of Membrane Science, Vol. 292, 2007, pp. 35-44. doi:10.1016/i.memsci.2007.01.007

[30] S. Y. Nam and J. R. Dorgan, "Non-Equuilibrium Nanobleds via Forced Assembly for Pervaporation Separation of Benzene from Cyclohexane: UNIFAQ-FV Group Contribution Caluculations," Journal of Memrane Science, Vol. 306, No. 1-2, 2007, pp. 186-195. doi:10.1016/j.memsci.2007.08.047

[31] S. J. Leu, J. S. Ou, C. H. Kuo, H. Y. Chen and T. H. Yang, "Pervaporation Separation of Azeotropic Mixtures in Polyurethane-Poly(Dimethylsiloxane) (PU-PDMS) Blend Membranes: Correlation with Sorption and Diffusion Behaviors in a Binary Solution System," Journal of Membrane Science, Vol. 347, 2010, pp. 108-115. doi:10.1016/j.memsci.2009.10.012 